\title{
Analytical and Experimental Evaluation of Piezoelectric Wafer Active Sensors Performances for Lamb Waves based Structural Health Monitoring in Composite Laminates
}

\author{
Giola S. Bottai ${ }^{1 \mathrm{a}}$, Nikos A. Chrysochoidis ${ }^{\mathrm{b}}$; Victor Giurgiutiu ${ }^{\mathrm{a}}$; and Dimitris A. Saravanos ${ }^{\mathrm{b}}$ \\ ${ }^{a}$ Department of Mechanical Engineering, University of South Carolina, 300 Main St. Columbia, SC \\ 29208 USA \\ ${ }^{\mathrm{b}}$ Department of Mechanical Engineering and Aeronautics University of Patras, Patras, GR 26500 \\ Greece
}

\begin{abstract}
Guided Lamb waves can be excited in composite materials through piezoelectric wafer active sensors (PWAS) to detect damage. PWAS are small, light-weight, inexpensive, and can be attached or embedded in composite structures. The proposed paper will present a parallel effort on two analytical approaches for predicting Lamb wave propagation in composite structures with surface attached PWAS. The first approach implements a layerwise mechanics theory and finite element for laminated composite beams with transducers and delaminations. The second approach uses a transfer matrix methodology (TM) and normal mode expansion (NME) to predict PWAS-plate interaction.

Wave propagation predictions are performed using 2-D layerwise beam theory approximating the in-plane displacement, the through-thickness displacements and the electrical field as a continuous assembly of linear layerwise fields through the thickness. The effect of delamination cracks can be predicted by the introduction of additional degrees of freedom. Prediction of symmetric, antisymmetric and shear horizontal Lamb wave dispersion curves is done for composite material structures using TM methodology developed by Nayfeh. NME technique is applied to predict the PWAS tuning curves on composite plates; theoretical and experimental results are compared. Prediction of sensor signals and local displacement curves through the thickness will be presented for composite structure.
\end{abstract}

Keywords: Structural health monitoring, Lamb wave, composite structures, piezoelectric sensors, laminate mechanics, delamination, finite element, tuning.

\section{Introduction}

Recent work on structural health monitoring (SHM) techniques has indicated benefits of using guided lamb waves in detecting various types of small size damage and in interrogating the condition of a structural component. The application of guided lamb waves towards the detection of damage in composite materials remains an open issue; hence, current interest is directed to the usage of lamb waves for revealing the damage in composite structures. A promising technique is the excitation and detection of lamb waves via piezoelectric wafer active sensors (PWAS), which are small, have low weight and cost and can be attached or embedded in composite structures. PWAS can excite all the possible Lamb wave modes at a given frequency - thickness product. However, SHM techniques, such as PWAS phased array technique and the time reversal process, relies on the capability to excite only one desired Lamb wave mode. In PWAS phased array applications, the assumption of the presence of a single low-dispersion Lamb wave mode (S0) is invoked. Since several Lamb wave modes traveling at different speeds would disturb the damage imaging results. It is important in Lamb wave application on composite plate, to be able to predict either the dispersion curves of the Lamb modes and the tuning frequency of the desired mode.

\footnotetext{
${ }^{1}$ Tel: (803) 7770619 Fax: (803) 7770106 bottai@engr.sc.edu
} 
The present paper presents two parallel efforts to cover the void in this area. The first is an analytical approach for the prediction of dispersion curves and tuning frequencies in laminated composite beams. The second is a mostly numerical approach for predicting the electromechanical response of composite beams with PWAS and delamination cracks.

\section{Lamb wave dispersion curves}

Lamb waves, or guided plate waves, are a type of ultrasonic waves that remain guided between two parallel free surfaces, such as the upper and lower surfaces of a plate or shell. Lamb wave theory is fully documented in a number of textbooks (Viktorov (2); Graff (3); Achenbach (4); Rose (5)). The wave equation in the case of an isotropic media can be expressed through two potential functions and the pressure and shear wave velocity characteristic of the material of the media. The shear horizontal wave propagation in this case is decoupled from the longitudinal (or pressure waves $\mathrm{P}$ ) and shear vertical (SV) wave propagations, and can be studied separately. For the case of a plate with free boundaries, the $\mathrm{P}$ and SV waves are coupled and their interaction is referred to as Lamb wave. Lamb waves modes can be symmetric and antisymmetric with respect to the through the thickness plane. The characteristic equation (RayleighLamb equation) of this type of waves is obtained by solving the wave equation and by applying stress free boundary conditions at the upper and lower surface. Fig. 1 shows the dispersion curves for an aluminum plate. The phase velocity changes with the frequency and the thickness of the material. For each material, there exists a threshold value, dependent by the material of the plate, below which only S0 and A0 modes exist.

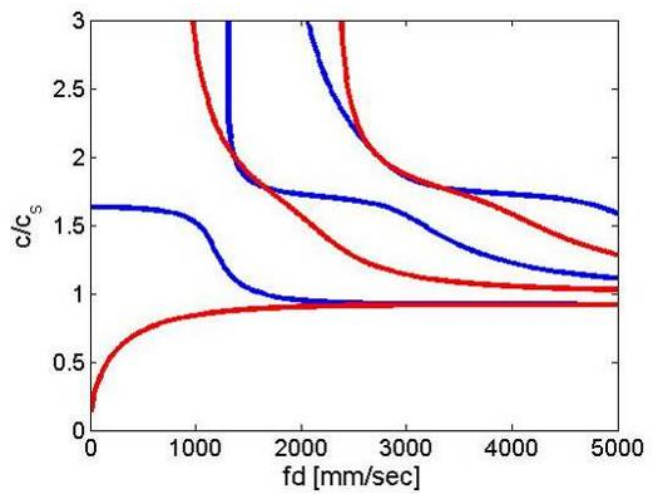

Fig. 1. Phase velocity for an aluminum plate.

The dispersion curves represented in the figures above are for real wavenumbers that corresponds to propagating modes.

In the case of composite materials it is not possible to find a close form solution of the dispersion curves, but there are different methods (Transfer matrix, Global matrix, and Stiffness matrix) that can be used to solve the problem. In this study the Nayfeh (6) method of the transfer matrix applied to anisotropic materials is used. For low frequency-thickness (fd) products, the method is stable. Other methods such as the Stiffness matrix have also proved to be stable and are more efficient.

Let consider composite plates made of layers of unidirectional fibers (Fig. 2).

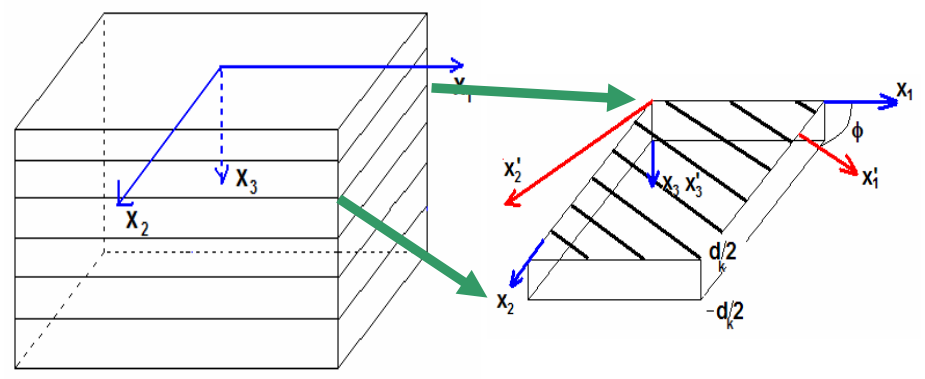

Fig. 2. $\mathrm{k}^{\text {th }}$ layer of a composite plate made of unidirectional layers.

The unidirectional layer in the matrix can have any orientation, the stiffness matrix in the global coordinate system is 


$$
C=T_{1}^{-1} C^{\prime} T_{2}
$$

where $T_{1}$ and $T_{2}$ are the transformation matrix. In the case of PWAS transducers, the wave propagation direction is $\theta=90^{\circ}$; the solution can be written as

$$
\left(u_{1}, u_{2}, u_{3}\right)=\left(U_{1}, U_{2}, U_{3}\right) e^{i \zeta\left(x_{1}+\alpha x_{3}-v t\right)}
$$

where $\zeta$ is the wavenumber, $v=\omega / \zeta$ is the phase velocity, $\omega$ is the circular frequency, $\alpha$ is an unknown parameter, and $U_{i}$ is the displacement amplitude.

Substituting equation (2) in the displacement equation leads to three coupled equations:

$$
\begin{aligned}
& \left(C_{11}+C_{55} \alpha^{2}-\rho v^{2}\right) u_{1}+\left(C_{16}+C_{45} \alpha^{2}\right) u_{2}+\left(C_{13}+C_{55}\right) \alpha u_{3}=0 \\
& \left(C_{16}+C_{45} \alpha^{2}\right) u_{1}+\left(C_{66}+C_{44} \alpha^{2}-\rho v^{2}\right) u_{2}+\left(C_{36}+C_{45}\right) \alpha u_{3}=0 \\
& \left(C_{13}+C_{55}\right) \alpha u_{1}+\left(C_{36}+C_{45}\right) \alpha u_{2}+\left(C_{55}+C_{33} \alpha^{2}-\rho v^{2}\right) u_{3}=0
\end{aligned}
$$

If the material coordinate and the global coordinate systems coincide, direction 2 is uncoupled from the other two. This means that the shear horizontal $(\mathrm{SH})$ wave is decoupled from the other two modes of propagation and the mathematical formulation is simpler. Following Nayfeh (6) formulation we obtain for each layer

$$
P_{k}=X_{k} D_{k} U_{k}
$$

Where $P$ is the displacement and stress vector $\left\{\left[u_{i}\right],\left[\sigma_{i 3}^{*}\right]\right\}, X$ is a $6 \times 6$ matrix, $U$ is the vector of the $U_{1 i}$ elements, and $D$ is the diagonal matrix whose elements are $e^{i \zeta\left(x_{1}+\alpha_{i} x_{3}-v t\right)}$. If we denote $A_{k}=X_{k} D_{k} X_{k}^{-1}$, it is possible to link the displacements and the stresses of the bottom layer to those of the top layer through equation (4):

$$
P_{k}^{+}=A_{k} P_{k}^{-}
$$

Applying the above procedure to each layer, the displacements and the stresses at the upper surface of the layered plate are related to the displacements and the stresses of the lower surface via the transfer matrix multiplication. The total transfer matrix expression is

$$
\left\{\begin{array}{l}
\left\{u^{+}\right\} \\
\left\{\sigma^{+}\right\}
\end{array}\right\}=\left[\begin{array}{ll}
{\left[A_{u u}\right]} & {\left[A_{u \sigma}\right]} \\
{\left[A_{u \sigma}\right]} & {\left[A_{\sigma \sigma}\right]}
\end{array}\right]\left\{\begin{array}{l}
\left\{u^{-}\right\} \\
\left\{\sigma^{-}\right\}
\end{array}\right\}
$$

In order to obtain the dispersion curve, we must impose stress free at the upper and lower surface boundaries:

$$
\left|A_{u \sigma}\right|=0
$$

We have developed this method on a MatLab program. The user enters a vector of the layer orientation, the program calculates the transfer matrix of each layer in the global coordinate system and then the total transfer matrix for different values of velocity and frequency. The program determines the velocity at which, for a given frequency, the matrix determinant change sign and then, by the method of bisection, finds the velocity solution.

A quasi-isotropic plate $\left[(0 / 45 / 90 /-45)_{2 s}\right]$ has been studied. The layer material is A534/AF252, same stiffness properties of T300/5208 (7). From the output values of the program it is possible to extract the first 3 modes of interest and convert the plot from wavenumber-thickness to frequency-thickness representation. From the phase velocity it is possible to calculate the group velocity curves. Fig. 3 shows the theoretical data and the experimental values. The experimental and theoretical data for the A0 mode are in quite good agreement; the experimental and theoretical data for the SH and S0 modes are also in good correlation with each other for low frequencies. At higher frequencies, where the two waves are closer, it is difficult to determine experimentally the wave location due to their superposition and dispersion. 


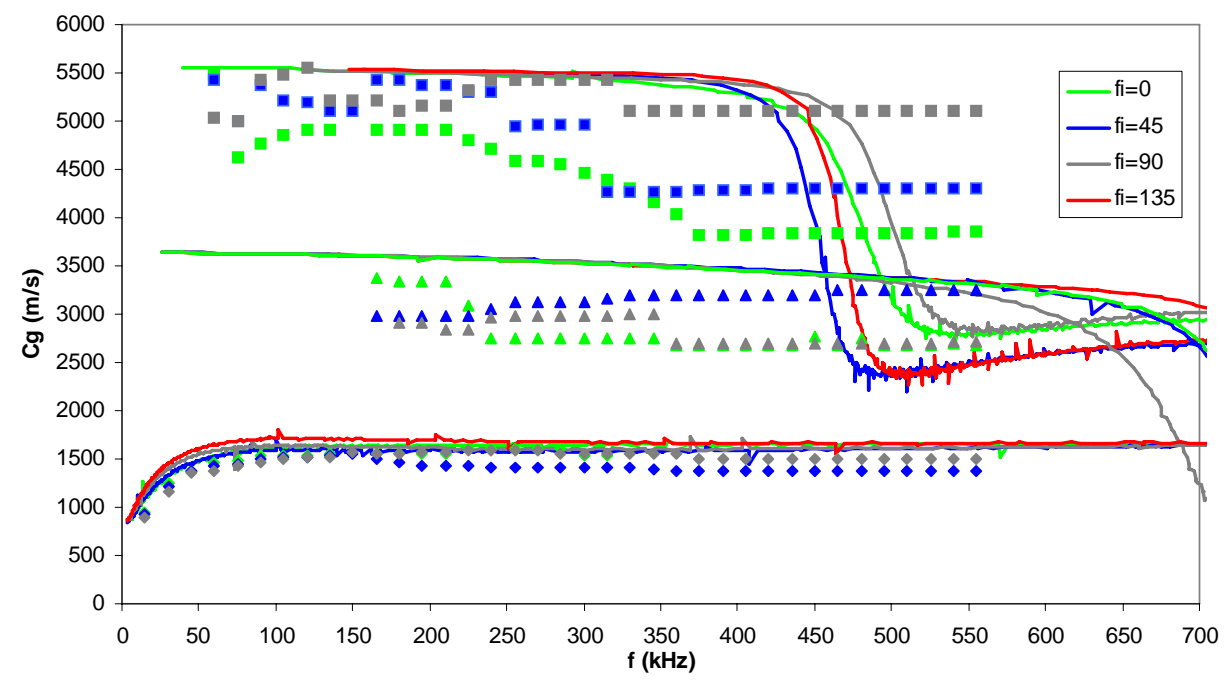

Fig. 3. Group velocities for a quasi isotropic plate. (solid lines)Theoretical values and (dots) experimental values.

\section{PWAS tuning on plates}

\subsection{Isotropic plates}

The PWAS, under electric excitation, transfers the oscillatory contractions and expansion to the bonded layer and the layer to the metal surface. In this process several factors influences the wave behavior: thickness of the bonding layer, geometry of the PWAS, thickness and material of the plate. The result of the influence of all these factors is the tuning of the PWAS with the material. This phenomenon has been studied by Giurgiutiu (8) who developed the theory of the interaction of the PWAS with the structure for a rectangular PWAS with an infinite dimension. The PWAS bonded on the structure is excited by a time-harmonic voltage. The expansion and contraction of the PWAS develop a timeharmonic interfacial shear stress $\tau(x) e^{i \omega t}$ between the structure and the PWAS. The shear stress acts only in the interval $|x|<a$. For thin layer of the bonded material $(1 \mu \mathrm{m})$, the shear stress is transmitted to the structure only at the ends of the PWAS. This is the ideal bonding solution, which can be considered true as a first approach to the problem. In this case the shear stress in the bonding layer can be assumed equal to:

$$
\left.\tau_{y x}(x)\right|_{y=h}=a \tau_{0}[\delta(x-a)-\delta(x+a)]
$$

where $\delta(x)$ is the Dirac function.

Having assumed a harmonic load distribution, the solution of the wave equation must be harmonic. The wave equation in terms of potential function is solved by the Fourier transform. In this way the displacement solution are found. The problem can be split into symmetric and antisymmetric solution for the case of isotropic plates. Once the integration constants have been derived, the displacement equations are transformed to the space domain with an inverse Fourier transformation. The inversion requires the use of the theorem of the residues. From the derived displacement equation it is derived the strain equation. Theoretical and experimental results have been compared by Bottai (9).

Raghavan and Cesnik (10) had extended this result to the case of a circular transducer. The results for the circular transducer are similar to the case of the strip PWAS. The resulting expression of the displacement has Bessel and Henkel function instead of sine and exponential functions as in the case of strip actuator.

\subsection{Composite plates}

$\mathrm{Xi}$ (11) has extended the integral transform solution derived for isotropic plates can be extended to the case of composite plates. The analytical solution of the inverse transform of the Fourier integral is in this case to be solved numerically with infinity of the integrand along the poles on the integral axis. A different method, NME, can be used to 
determine the transducer frequencies for any kind of plates. It has been used for application as hollow cylinders excited by wedge transducers where the dispersion curves have a second order of infinity (12) (13), but so far I have not seen any application for composite plates.

In NME the fields generated in the structure due to the application of the surface loading are expanded in the form of an infinite series of the normal modes of the structure itself. For complete mathematical rigor of the NME method, it must be proofed that the field distribution of mode set is complete and orthogonal. We can assume that the set of acoustic waveguide mode functions is complete (Auld (12)).

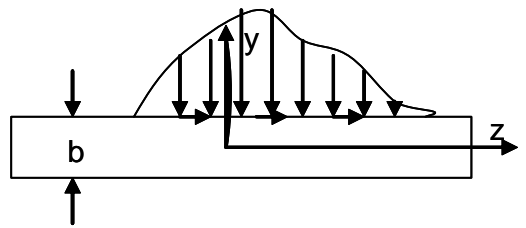

Fig. 4. Plate subject to a surface traction

Following Auld (14) and Rose (5) we consider a plate subject to a external traction $\boldsymbol{t}(\eta)$ exerted by the actuator (see Fig. 4), the acoustic field in the plate can be expressed as:

$$
\mathbf{v}_{1}(y, z)=\sum_{m} a_{m}(z) \mathbf{v}_{m}(y)
$$

where

$$
a_{ \pm n}(z)=\frac{e^{\mp i \beta_{n} z}}{4 P_{n n}} \tilde{\mathbf{v}}_{ \pm n}\left(\frac{b}{2}\right) \cdot \int_{-\infty}^{\infty} e^{ \pm i \beta_{n} \eta} \mathbf{t}(\eta) d \eta
$$

And $P_{n n}$ is the average power flow of the $n^{\text {th }}$ mode in the $z$ direction per unit waveguide width (in the $x$ direction).

In the case of ideal bonding solution between the PWAS and the plate, the shear stress in the bonding layer is concentrated at the ends (13). In this case the amplitude $a_{n}$ can be written as:

$$
a_{ \pm n}(z)=i a \tau_{0} \frac{\tilde{v}_{n z}(b / 2)}{2 P_{n n}} \sin \beta_{n} a e^{\mp i \beta_{n} z}
$$

Knowing the amplitude it is possible to derive the strain field for any wave mode. Chapter 5 will present experimental and theoretical comparison for dispersion curves and PWAS tuning on unidirectional composite plates.

\section{Delaminated Layerwise Formulation}

A layerwise formulation and a finite element approach were developed by Chysochoidis and Saravanos (13) assuming a piecewise linear transverse displacements field through the laminate thickness. This finite element based model has the ability to describe the electromechanical response of delaminated composite beams with piezoelectric actuators and sensors. Key elements of the formulation are briefly described herein.

\subsection{Theoretical Formulation}

Governing Material Equations. Each ply composite or piezoelectric is assumed to follow linear constitutive equations of piezoelectricity:

$$
\begin{array}{ll}
\sigma_{i}=C_{i j}^{E} S_{j}-e_{i k} E_{k} & i, j=1, \ldots, 6 \\
D_{m}=e_{m j} S_{j}+\varepsilon_{m k}^{S} E_{k} & k, m=1, \ldots, 3
\end{array}
$$

$\sigma_{i}$ and $S_{j}$ are the mechanical stresses and engineering strains in vectorial notation; $E_{m}$ is the electric field vector; $D_{m}$ is the electric displacement vector; $\mathrm{C}_{\mathrm{ij}}, \mathrm{e}_{\mathrm{lk}}$ and $\varepsilon_{\mathrm{mk}}$ are the elastic stiffness; piezoelectric and electric permittivity tensors 
respectively. Superscripts $\mathrm{E}$ and $\mathrm{S}$ indicate constant electric field and strain conditions, respectively. The above equations encompass the behavior of an off-axis homogenized fibrous piezocomposite layer.

Equation of Equilibrium. The variational statement of the equations of equilibrium for the piezoelectric structure is,

$$
\int_{\mathrm{A}}-\delta \mathrm{HdA}+\int_{\mathrm{A}} \delta \underset{\sim}{\mathrm{u}}(-\rho \underset{\sim}{\ddot{u}}) \mathrm{dA}+\oint_{\Gamma_{\tau}} \delta \underline{\sim}^{\mathrm{T}} \bar{\tau} \mathrm{d} \Gamma+\oint_{\Gamma_{\mathrm{D}}} \delta \bar{\varphi} \overline{\mathrm{D}} \mathrm{d} \Gamma=0
$$

where $A$ is the $x-z$ surface of the beam; $H_{L}$ is the electric enthalpy of the piezoelectric laminate; $\bar{\tau}$ and $\bar{D}$ are, respectively, the surface tractions and charge, acting on the boundary surface $\Gamma_{\tau}, \Gamma_{D} ; \underset{\sim}{u}=\left\{\begin{array}{c}u \\ w\end{array}\right\}$ is the displacement vector.

Kinematic Assumptions. A typical laminate is assumed to be subdivided into $\mathrm{N}$ discrete layers, where each discretelayer may contain either a single ply, or a sub-laminate, or a sub-ply. The damage, consisting of either a single or multiple delamination cracks is considered to exist at the interfaces of two adjacent plies. Piecewise linear in-plane, transverse displacement and electric potential fields are assumed through the laminate thickness, which maintains continuity across the discrete layer boundaries, yet, allows for different slopes within each discrete layer, and admit sliding and opening across a delamination crack. In this manner, the displacement field (schematically shown in Figure 5) of the laminate, taking into account $\mathrm{N}_{\mathrm{d}}$ delamination cracks takes the form:

$$
\begin{gathered}
\mathrm{u}(\mathrm{x}, \mathrm{z})=\sum_{\mathrm{i}=1}^{\mathrm{N}}\left(\mathrm{u}^{\mathrm{i}}(\mathrm{x}) \psi_{1}^{\mathrm{i}}\left(\mathrm{z}_{\mathrm{i}}\right)+\mathrm{u}^{\mathrm{i}+1}(\mathrm{x}) \psi_{2}^{\mathrm{i}}\left(\mathrm{z}_{\mathrm{i}}\right)\right)+\sum_{\mathrm{k}=1}^{\mathrm{Nd}} \tilde{\mathrm{u}}^{\mathrm{k}}(\mathrm{x}) \psi^{\mathrm{k}}\left(\mathrm{z}_{\mathrm{k}}\right) \mathrm{H}\left(\mathrm{z}-\mathrm{z}_{\mathrm{k}}\right) \\
\mathrm{w}(\mathrm{x}, \mathrm{z})=\sum_{\mathrm{i}=1}^{\mathrm{N}}\left(\mathrm{w}^{\mathrm{i}}(\mathrm{x}) \psi_{1}^{\mathrm{i}}\left(\mathrm{z}_{\mathrm{i}}\right)+\mathrm{w}^{\mathrm{i}+1}(\mathrm{x}) \psi_{2}^{\mathrm{i}}\left(\mathrm{z}_{\mathrm{i}}\right)\right)+\sum_{\mathrm{k}=1}^{\mathrm{Nd}} \tilde{\mathrm{w}}^{\mathrm{k}} \psi^{\mathrm{k}}\left(\mathrm{z}_{\mathrm{k}}\right) \mathrm{H}\left(\mathrm{z}-\mathrm{z}_{\mathrm{k}}\right) \\
\varphi(\mathrm{x}, \mathrm{z})=\Phi^{\mathrm{i}}(\mathrm{x}) \psi^{\mathrm{i}}\left(\mathrm{z}_{\mathrm{i}}\right)+\Phi^{\mathrm{i}+1}(\mathrm{x}) \psi^{\mathrm{i}+1}\left(\mathrm{z}_{\mathrm{i}}\right)
\end{gathered}
$$

where superscripts $\mathrm{i}=1, \ldots, \mathrm{N}$ indicate the discrete layers, $\mathrm{k}=1, \ldots, \mathrm{N}^{\mathrm{d}}$ the number of the delaminations through the thickness. At equations (14) and (15) terms $\mathrm{u}^{\mathrm{i}}, \mathrm{u}^{\mathrm{i}+1}$ and $\mathrm{w}^{\mathrm{i}}, \mathrm{w}^{\mathrm{i}+1}$ are the axial and transverse displacements respectively at the interfaces of each discrete layer effectively describing the extension, rotation and through-thickness stretching, of the layer. Also $\tilde{\mathrm{u}}^{\mathrm{k}}$ and $\tilde{\mathrm{w}}^{\mathrm{k}}$ are the new degrees of freedom describing the sliding and opening across the faces of the $\mathrm{k}^{\text {th }}$ delamination (relative displacements). $\mathrm{H}$ is the Heaviside's step function and $\mathrm{z}_{\mathrm{k}}$ is the distance of the $\mathrm{k}^{\text {th }}$ delamination from the mid-plane.

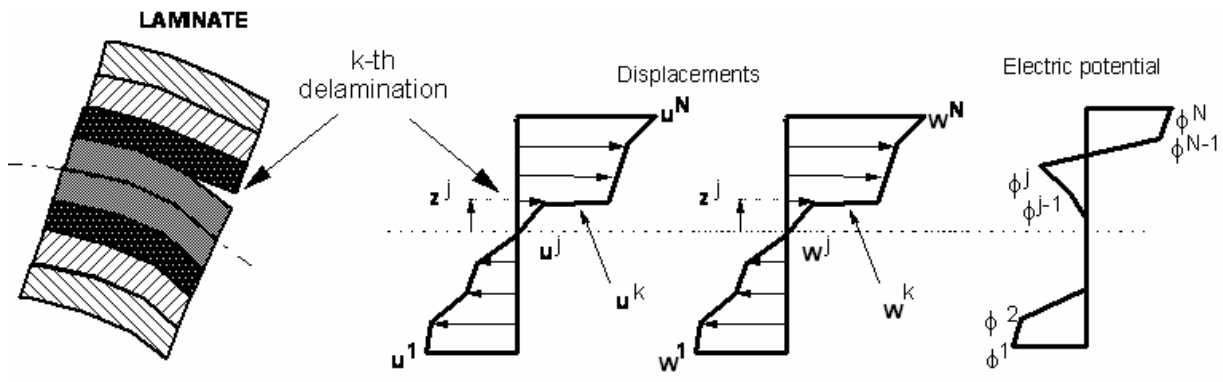

(a) Smart Laminate

(b) Discrete-layer laminate theory assumptions

Fig. 5. Illustration of the assumed displacement and electrical fields through the thickness of a delaminated composite laminate 
Finally, $\Psi^{\mathrm{i}}$ and $\Psi^{\mathrm{k}}$ are the linear interpolation functions through the layer thickness and $\mathrm{z}_{\mathrm{i}}$ is the thickness coordinates of layer $i$ and $z_{k}$ the thickness of the layer above the $k^{\text {th }}$ crack. Also $\varphi$ is the electric potential and $\Phi^{i}$ and $\Phi^{i+1}$ are the potentials at interfaces of each layer.

Generalized strain and electric field relations. The kinematic assumptions in eqs. (14-15) yield in each discrete layer an axial, transverse and shear strains $\mathrm{S}_{1}, \mathrm{~S}_{3}$ and $\mathrm{S}_{5}$ and an axial and transverse electrical field component of the form,

$$
\begin{aligned}
& \mathrm{S}_{1}=\sum_{\mathrm{i}=1}^{\mathrm{N}}\left(\mathrm{u}_{, x}^{\mathrm{i}} \psi_{1}^{\mathrm{i}}+\mathrm{u}_{, \mathrm{x}}^{\mathrm{i}+1} \psi_{2}^{\mathrm{i}}\right)+\sum_{\mathrm{k}=1}^{\mathrm{Nd} d} \tilde{\mathrm{u}}_{, \mathrm{x}}^{\mathrm{k}} \psi^{\mathrm{k}} \mathrm{H}\left(\mathrm{z}-\mathrm{z}_{\mathrm{k}}\right) \\
& \mathrm{S}_{3}=\sum_{\mathrm{i}=1}^{\mathrm{N}}\left(\mathrm{w}^{\mathrm{i}} \psi_{1, \zeta_{1}}^{\mathrm{i}} \frac{2}{\mathrm{hi}}+\mathrm{w}^{\mathrm{i}+1} \psi_{2, \zeta_{1}}^{\mathrm{i}} \frac{2}{\mathrm{hi}}\right)+\sum_{\mathrm{k}=1}^{\mathrm{Nd}}\left(\tilde{\mathrm{w}}^{\mathrm{k}} \psi_{, \zeta_{1}}^{\mathrm{k}} \frac{2}{\mathrm{hi}} \mathrm{H}\left(\mathrm{z}-\mathrm{z}_{\mathrm{k}}\right)+\hat{\mathrm{w}}^{\mathrm{k}} \psi^{\mathrm{k}} \hat{\delta}\left(\mathrm{z}-\mathrm{z}_{\mathrm{k}}\right)\right)
\end{aligned}
$$

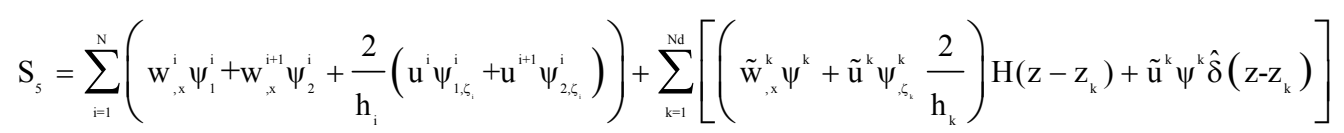

$$
\begin{aligned}
& \mathrm{E}_{1}=\sum_{\mathrm{i}=1}^{\mathrm{N}}\left(-\left(\Phi_{, \mathrm{x}}^{\mathrm{i}} \Psi_{1}^{\mathrm{i}}+\Phi_{, \mathrm{x}}^{\mathrm{i}+1} \Psi_{2}^{\mathrm{i}+1}\right)\right) \\
& \mathrm{E}_{3}=\sum_{\mathrm{i}=1}^{\mathrm{N}}\left(-\left(\Phi^{\mathrm{i}} \Psi_{{ }_{\zeta_{\mathrm{k}}}}^{\mathrm{i}}+\Phi^{\mathrm{i}+1} \Psi_{{ }_{\zeta_{\mathrm{k}}}}^{\mathrm{i}+1}\right) \frac{2}{\mathrm{~h}_{\mathrm{k}}}\right)
\end{aligned}
$$

where $\hat{\delta}$ is the Dirac impulse function. In the above strain equations (17) to (20), $\mathrm{u}_{, \mathrm{x}}^{\mathrm{i}}, \mathrm{u}^{\mathrm{i}+1}{ }_{, \mathrm{x}}, \mathrm{w}_{, \mathrm{x}}^{\mathrm{i}}, \mathrm{w}^{\mathrm{i}+1}{ }_{, \mathrm{x}}$ represent $\mathrm{S}_{1}$ and $\mathrm{S}_{3}$ strain contributions, respectively, within each discrete layer of a healthy laminate, whereas, $\mathrm{u}_{, \mathrm{x}}^{\mathrm{k}}$ and $\mathrm{w}^{\mathrm{k}}{ }_{\mathrm{x}}$ expresses effect of the $\mathrm{k}^{\text {th }}$ delamination on the axial and transverse strains $\mathrm{S}_{1}$ and $\mathrm{S}_{3}$ above the crack. In the shear strain the sum of the first two terms yields a constant shear strain term through the layer and the last two the effect of delamination. Equation (21) offers a constant variation in the electrical field in each discrete layer. The previous strain and electrical field equations were included into the variational form of equilibrium equations, and the equivalent laminate stiffness and mass matrices were derived.

Finite Element Formulation. A 2 node beam finite element was formulated based upon on the previous laminate mechanics with linear shape functions $\mathrm{N}(\mathrm{x})$ used for the in plane, interlaminar displacements and electric potentials. In this manner, the local approximations of the generalized state variables in the element take the form:

$$
\begin{aligned}
& \left\{\mathrm{u}^{\mathrm{i}}(\mathrm{x}, \mathrm{t}), \tilde{\mathrm{u}}^{\mathrm{k}}(\mathrm{x}, \mathrm{t}), \mathrm{w}^{\mathrm{i}}(\mathrm{x}, \mathrm{t}), \tilde{\mathrm{w}}^{\mathrm{k}}(\mathrm{x}, \mathrm{t})\right\}=\sum_{\mathrm{i}=1}^{\mathrm{L}}\left\{\mathrm{u}^{\mathrm{im}}(\mathrm{t}), \tilde{\mathrm{u}}^{\mathrm{km}}(\mathrm{t}), \mathrm{w}^{\mathrm{im}}(\mathrm{t}), \tilde{\mathrm{w}}^{\mathrm{km}}(\mathrm{t})\right\} N^{\mathrm{i}}(\mathrm{x}) \\
& \Phi^{\mathrm{k}}(\mathrm{x})=\sum_{\mathrm{i}=1}^{L} \Phi^{\mathrm{ki}} N^{\mathrm{i}}(\mathrm{x})
\end{aligned}
$$

where $\mathrm{i}=1, \ldots, \mathrm{N}+1$ and $\mathrm{k}=1, \ldots, \mathrm{N}^{\mathrm{d}}$ (maximum number of delaminations that exist through the thickness of the element); $\mathrm{L}$ denotes the element length, for a 2-node $L=2$ beam element. Substituting the above equations into the governing equations of equilibrium which are expressed in variational form (13), the coupled piezoelectric system can be expressed in a discrete matrix form:

$$
\left[\begin{array}{cc}
{\left[\mathrm{M}_{\mathrm{uu}}\right]} & 0 \\
0 & 0
\end{array}\right]\left\{\begin{array}{c}
\{\ddot{\mathrm{u}}\} \\
\left\{\varphi^{\mathrm{s}}\right\}
\end{array}\right\}+\left[\begin{array}{cc}
{\left[\mathrm{K}_{\mathrm{uu}}\right]} & {\left[\mathrm{K}_{\mathrm{u \varphi}}^{\mathrm{FF}}\right]} \\
{\left[\mathrm{K}_{\varphi \mathrm{Fu}}^{\mathrm{FF}}\right]} & {\left[\mathrm{K}_{\varphi \varphi}^{\mathrm{FF}}\right]}
\end{array}\right]\left\{\begin{array}{c}
\{\overline{\mathrm{u}}\} \\
\left\{\varphi^{\mathrm{F}}\right\}
\end{array}\right\}=\left\{\begin{array}{c}
\{\mathrm{P}\}-\left[\mathrm{K}_{\mathrm{u \varphi}}^{\mathrm{FA}}\right]\left\{\varphi^{\mathrm{A}}\right\} \\
\left\{\mathrm{Q}^{\mathrm{F}}\right\}-\left[\mathrm{K}_{\varphi \varphi}^{\mathrm{FA}}\right]\left\{\varphi^{\mathrm{A}}\right\}
\end{array}\right\}
$$


Submatrices $\left[\mathrm{K}_{\mathrm{uu}}\right],\left[\mathrm{K}_{\mathrm{u} \varphi}\right]$ and $\left[\mathrm{K}_{\varphi \varphi}\right]$ indicate the total elastic, piezoelectric and permittivity matrices of the beam structure; $\left[\mathrm{M}_{\mathrm{uu}}\right]$ indicates the mass matrix; material, structural and delamination parameters are included in the previous sublaminates. Superscripts F and A indicate, respectively, sensory (free) and active (applied) electric potential components; $\{\mathrm{P}\}$ is the applied mechanical forces vector and $\left\{\mathrm{Q}^{\mathrm{F}}\right\}$ is the applied electric charge at the sensors. The previously described electromechanical system using the Newmark transient methodology is used to predict the time response of delaminated composite beams.

\subsection{Results and Discussion}

This section presents numerical results predicted by the present model for two different materials. The first one is an isotropic aluminium beam having dimensions: $0.4 \times 0.002 \mathrm{~m}$. The second case is two Carbon/Epoxy composite beams with fiber volume ratios in the range of $0.57-0.63,1.27 \mathrm{~mm}$ nominal ply thicknesses, $280 \mathrm{~mm}$ length and $25 \mathrm{~mm}$ width. The first beam is pristine and the second has a full width delamination crack covering the $10 \%$ of the total beam length. In both cases on the beams surface two piezoceramic patches (PZ27) are modeled, the first one as actuator and the second as sensor. Position of piezoceramics for healthy or damaged beams as long as the delamination position is shown at the fig 6 .

Prediction of Lamb modes requires appropriate positions for displacement predictions. Fig $7 \mathrm{a}$ and $\mathrm{b}$ present the displacement field through the thickness of symmetric (S0) and antisymmetric (A0) lamb waves. According to these plots time waveforms of the transverse displacements are simulated for the points indicated at the Fig. 8, which are the positions, were the displacement field reaches maxima. In order to excite these waves we used a 7 cycles tone burst, a narrowband actuation signal choosing various central frequencies.

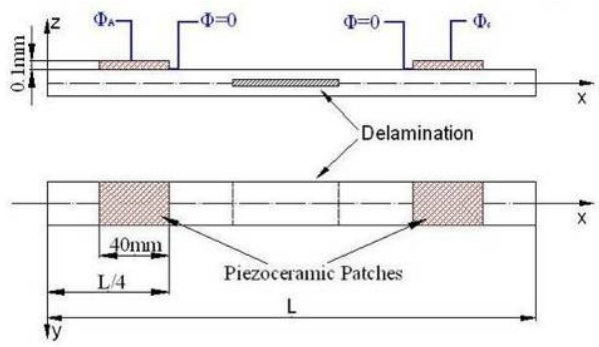

Fig 6. Delaminated composite beam with piezoceramic patches

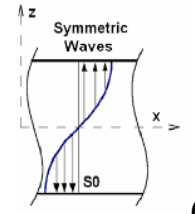

(a)

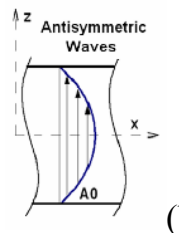

(b)

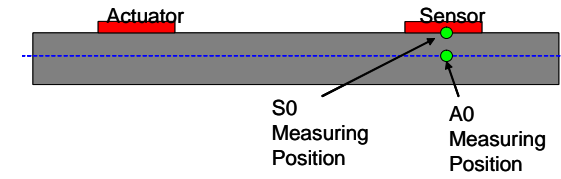

Fig.8. Positions were w predictions mostly capture transverse So and Ao Lamb waves, respectively

Aluminum Beam. The transient response of the aluminum beam described above is predicted under free-free supporting conditions. The pseudo Wigner-Ville distributions (PWVD) are extracted via the time waveforms and illustrate the frequency content of the given signals as a function of the time. Predictions were obtained for two different central frequencies, $10 \mathrm{KHz}$ and $1 \mathrm{MHz}$, of a tone burst voltage signal applied at the actuator. Figures 9 and 10 illustrate the dispersion curves simulating the time response of an aluminum beam subjected to such narrowband actuation signals. Each one of these figure entails three sub-figures. The top one presents time response prediction of the finite element model, the left the power spectrum and finally the contoured the pseudo Wignel-Ville distribution. Dispersion curves present the ability of the developed finite element model to simulate the propagation of symmetric and antisymmetric lamb waves having modal frequencies near the actuation frequency. These PWVD describe the change at frequency content of these waves as a function of time. 

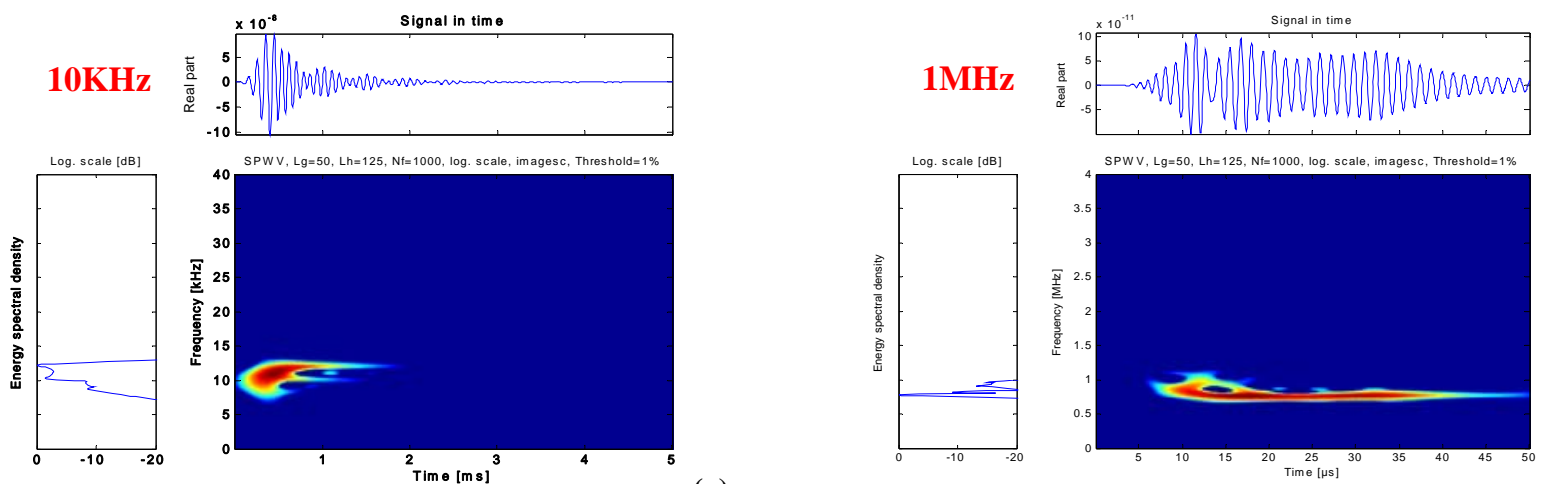

(a)

Fig. 9 Antisymmetric transverse dispersion curves for (a) actuation at $10 \mathrm{KHz}$ and (b) actuation at $1 \mathrm{MHz}$

$10 \mathrm{KHz}$
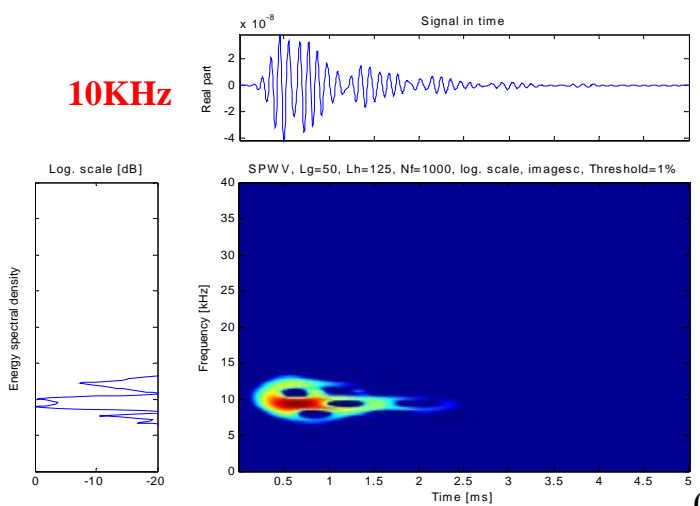

$1 \mathrm{MHz}$
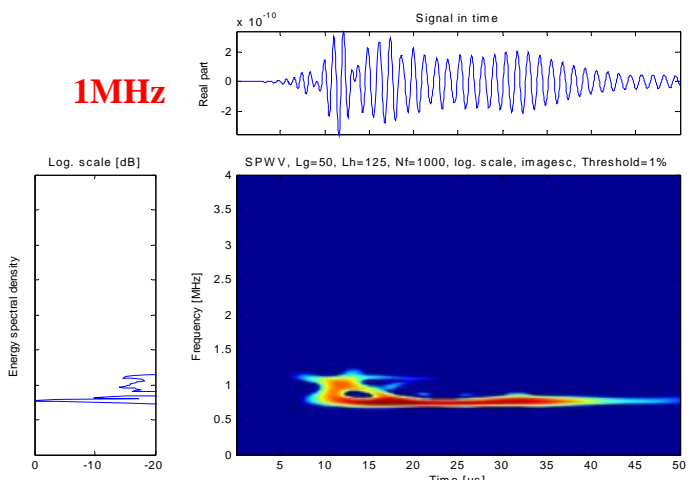

(b)

Fig. 10 Symmetric transverse dispersion curves for: (a) actuation at $10 \mathrm{KHz}$ and (b) actuation at $1 \mathrm{MHz}$

Delaminated Carbon/Epoxy Beam. The effect of delamination on predicted PWVD curves was further investigated for a carbon epoxy composite beam. The beams were modeled under free-free supporting conditions with two piezoceramic patches, an actuator and a sensor as previously described. Time waveforms were predicted, for the pristine and delaminated beam respectively, using a $200 \mathrm{KHz}$ tone burst actuation signal. Some of the obtained dispersion curves of the symmetric transverse displacement fields are shown in Figure 11. Comparing the two plots, between a healthy and a delaminated composite beam having a crack equal to $10 \%$ of its length, we observe that the delamination drastically affects the predicted response. These results lend credence to the capability of the developed finite element model, which assumes variable transverse displacement field, to simulate the propagation of transverse lamb waves. Based on observed results, monitoring transverse displacements, under such lamb waves, seems to provide a good damage indicator appearing to be very sensitive even to small delamination sizes. Axial displacements were found to be less sensitive. Additionally, Figure 12 compares the predicted dispersion curves of simulated sensory voltage waveforms. Sensory voltage seems to be less sensitive, compared to transverse displacements, to the presence of delamination. They are many possible reasons for this lower sensitivity, one is the effect of sensor size on the sensor signal for a given excitation frequency and wavelength; another is the low contribution of transverse strains on the direct piezoelectric effect of the given sensor configuration. Both are presently under investigation and will be addressed in a future study. 

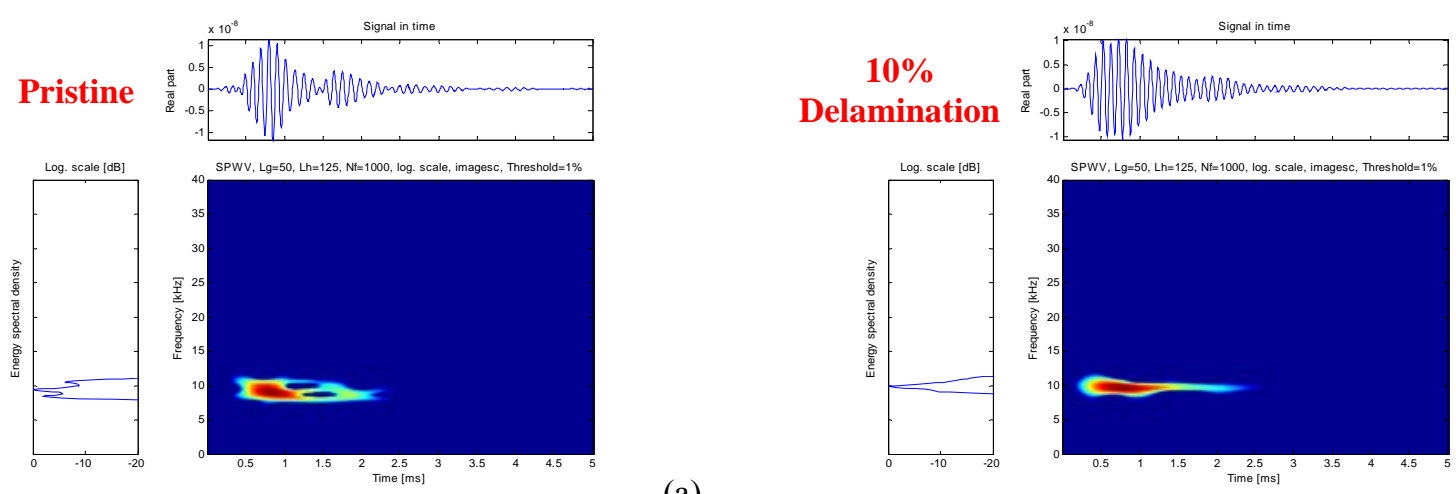

(b)

Fig.11. Dispersion curves of symmetric transverse displacements (a) without and (b) with a delamination covering $10 \%$ of the total length
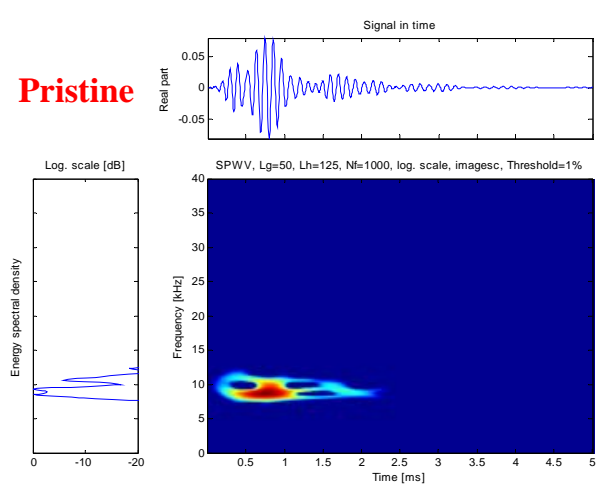

(a)

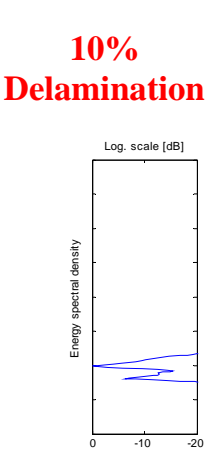

Fig 12. Dispersion curves of predicted sensory voltage (a) without and (b) with a delamination covering $10 \%$ of the total length.

\section{Dispersion curves and PWAS tuning on composite plate}

Pitch-catch experiments were performed on a composite plate in which one PWAS served as transmitter and another PWAS served as receiver. 10 round PWAS diameter $7 \mathrm{~mm}, 0.2 \mathrm{~mm}$ thick (American Piezo Ceramics APC-850) where installed. PWAS \#1 was used as the transmitter, while the other nine were used as receivers. In the present paper only the data received by PWAS \#3 on $90^{\circ}$ fiber direction are analyzed. Fig. 13 shows the PWAS configuration on the composite plate. Clay was used to attenuate the reflection from the boundary.
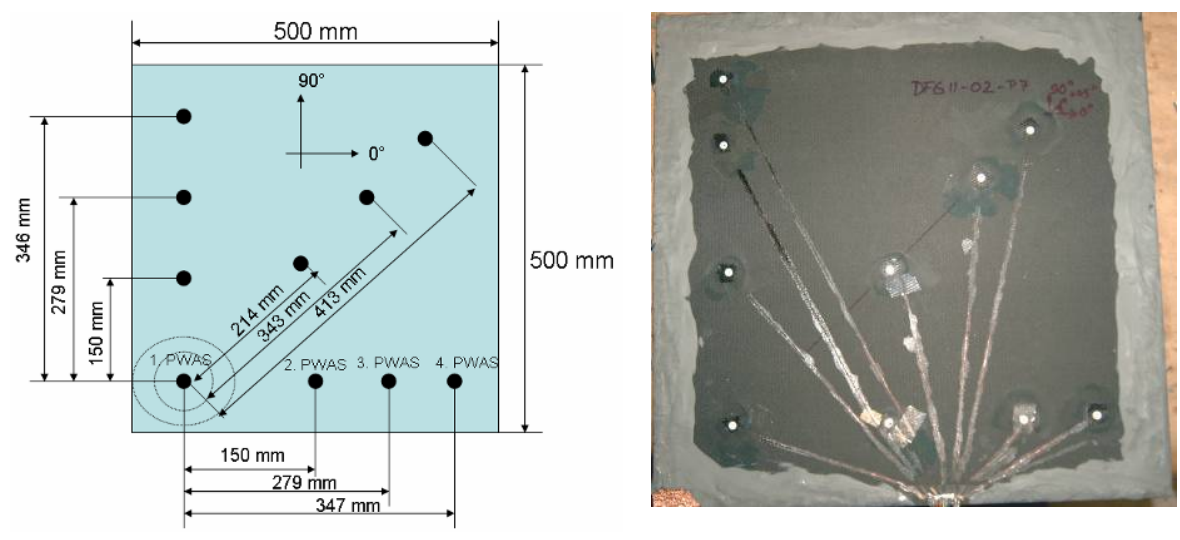

Fig. 13. PWAS configuration on unidirectional composite plate 
During the experiments two waves were detected, S0 and A0 modes. The SH0 mode was not possible to be located. The experiments were performed without an amplifier. The S0 amplitude was always quite low and at frequency above 140 $\mathrm{kHz}$, the wave amplitude was of the order of magnitude of the noise.

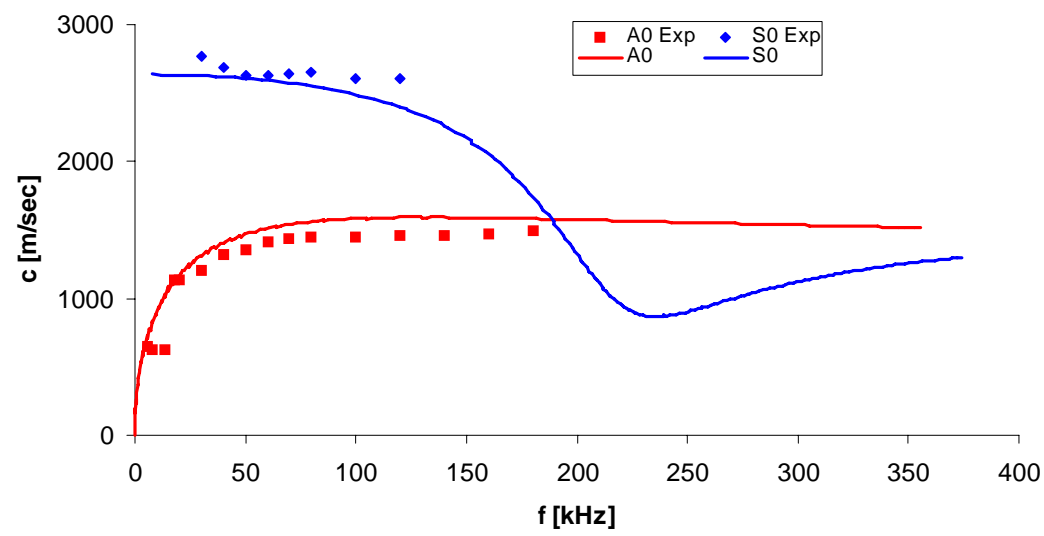

Fig. 14. Group velocity dispersion curves. Theoretical results (solid lines) and experimental data (dot).

Fig. 14 shows the group velocity for the S0 and A0 mode. The dot lines are the experimental data. The wave packets were detected up to $185 \mathrm{kHz}$. Beyond that frequency it was not possible to distinguish the wave packet from the noise. The Neyfeh theory implemented on MatLab was used to predict the dispersion curves. The figure reports only the S0 and A0 modes. The theoretical values are in good agreement with the experimental results.

The experimental data were used to construct the tuning curves. Fig. 15 shows the experimental data. The ordinate axis unit in the case of experimental results is $\mathrm{mV}$. The NME theory was used to derive the theoretical prediction of the tuning curves. Both $\mathrm{A} 0$ and $\mathrm{S} 0$ modes were scaled of the same amount to compare them to the experimental data.

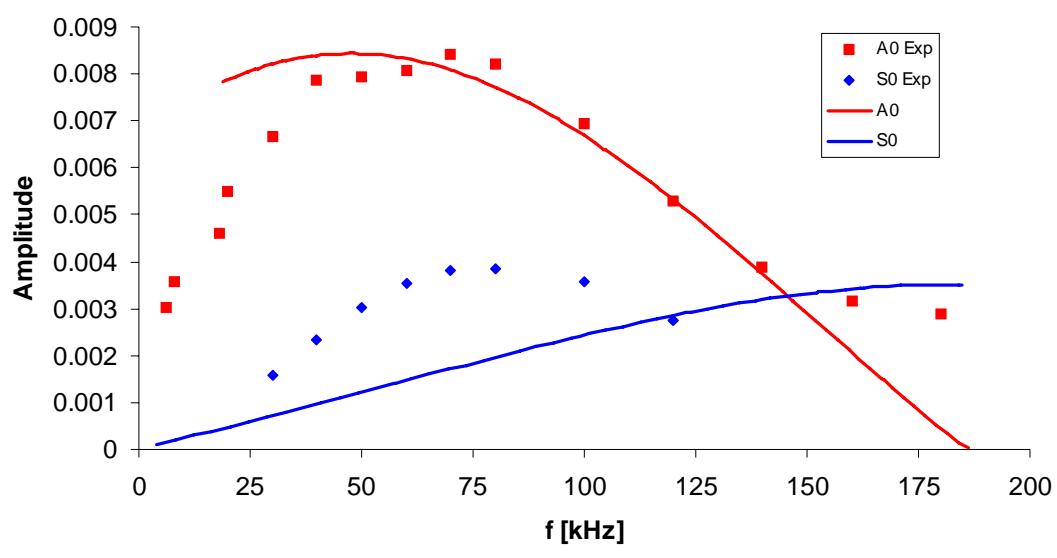

Fig. 15. Tuning curves. Theoretical results (solid lines) and experimental data (dot).

The A0 mode tuning curve predicts with good accuracy the frequency at which the A0 amplitude reaches a maximum. For the S0 mode, the theoretical and experimental results are not in agreement. Further investigation on different transmitter - receiver distance and direction with the reference to the fiber direction must be investigated in order to determine the validity of the method presented here.

\section{Conclusions}

A transfer matrix method has been presented where the exact solution is derived for each ply of the anisotropic plate. This method capable to predict the dispersion curves for composite plates made of several plays with different fiber direction, and different ply material. Through the derivation of the dispersion curved it is possible to derive the tuning frequency for a PWAS installed on a composite plate. The method used is the NME, the prediction for A0 are useful to 
locate the maximum of the mode under consideration. The prediction for S0 mode was not in accordance with the experimental results. However, being quite difficult to locate the experimental S0 wave because of its low amplitude with respect to the noise level, the authors will further investigate this discrepancy with more experiments on unidirectional composite plates.

A delaminated electromechanical layerwise formulation was developed assuming a variable transverse displacements field. A beam finite element was further developed and used to predict time response of the transverse displacements and sensory voltage. Pseudo Wignel-Ville distributions of the predicted time waveforms were presented illustrating beam model dispersion curves at various frequencies. Predictions were obtained for healthy isotropic and delaminated composite beams. Predictions proved the performance of the developed model to simulate the propagation of lamb waves having frequencies close to the actuation. Also these predictions illustrate the changes at the frequency content of the time waveform as a function of time. As a damage indicator, observations of the transverse displacements seemed to be very sensitive to reveal the existence even of small delamination sizes. On the other hand sensory voltage seems to be less sensitive and its ability to reveal the damage requires further investigation.

\section{Acknowledgment}

The financial support of National Science Foundation award \# CMS 0408578, Dr. Shih Chi Liu, program director is gratefully acknowledged.

\section{References}

1. Kudva, J. N.; Marandis, C.; Gentry, J. (1993) "Smart Structures Concepts for Aircraft Structural Health Monitoring”, SPIE Vol. 1917, pp. 964-971, 1993

2. Viktorov I. A. (1967) "Rayleigh and Lamb waves”, Plenum Press, New York, 1957

3. Graff K. F. (1991) "Wave motion in elastic solids", Dover Publications, inc, New York, 1991

4. Achenbach J. D., (1999) "Wave propagation in solids", North-Holland, 1999Graff K. F. (1991) Wave motion in elastic solids, Dover Publications inc. 1991

5. Rose J. L. (1999) "Ultrasonic waves in solid media”, Cambridge University Press, 1999

6. Nayfeh A. H., (1995) "Wave propagation in layered anisotropic media with application to composites" Elsevier, 1995

7. Herakovich C. T. (1998) “Mechanics of Fibrous Composites”, John Wiley \& Sons, Inc 1998

8. Giurgiutiu, V. (2005) "Tuned Lamb-Wave Excitation and Detection with Piezoelectric Wafer Active Sensors for Structural Health Monitoring", Journal of Intelligent Material Systems and Structures, Vol. 16, No. 4, pp. 291-306, 2005

9. Bottai G., Giurgiutiu V.; "Simulation of the Lamb wave interaction between piezoelectric wafer active sensors and host structure"; SPIE's $12^{\text {th }}$ International Symposium on Smart Structures and Materials and $10^{\text {th }}$ International Symposium on NDE for Health Monitoring and Diagnostics, Sensors and Smart Structures Technologies for Civil, Mechanical, and Aerospace Systems Conference, San Diego, CA, 7-10 March, 2005, paper \# 5765-29

10. Raghavan A., Cesnik C. E. S.; (2004) "Modeling of piezoelectric-based Lamb-wave generation and sensing for structural health monitoring"; Smart Structures and Materials July 2004, pp. 419-430

11. Xi L. (2002) "Elastic waves in anisotropic laminates", CRC Press, 2002

12. Ditri J. J., Rose J. L. (1992) "Excitation of guided elastic wave modes in hollow cylinders by applied surface tractions", Journal of Applied Physics, 72 (7) October 1992

13. Li J., Rose J. L. (2001) "Excitation and propagation of non-axisymmetric guided waves in a hollow cylinder", Journal of Acoustic Society of American, 109 (2) February 2001

14. Auld B. A. (1990) "Acoustic Fields and waves in solids", John Wiley \& Son Vol. 1 and 2

15. Chrysochoidis N.A., Saravanos D.A.; "2D Layerwise Modeling of High-Frequency Modal Response in Delaminated Composite Beams with Active Piezoelectric Sensors", $3^{\text {rd }}$ European conference on structural health monitoring, Granada, Spain, 5-7 July, 2006 